\title{
Use of ultrasound as the initial screening exam for hepatocellular carcinoma in high risk populations
}

\author{
Jason B. Welch ${ }^{1}$, Richa Shukla ${ }^{1}$, Nnenna Igbo ${ }^{1}$, Ashwani K. Singal ${ }^{2}$, Joao Kazan-Tannus ${ }^{3}$, \\ Roger D. Soloway ${ }^{2}$ \\ ${ }^{1}$ Department of Internal Medicine, University of Texas Medical Branch, Galveston, USA \\ ${ }^{2}$ Department of Internal Medicine, Division of Gastroenterology, University of Texas Medical Branch, Galveston, USA \\ ${ }^{3}$ Department of Radiology, University of Texas Medical Branch, Galveston, USA \\ Email: jaswelch@utmb.edu
}

Received 22 February 2013; revised 27 March 2013; accepted 6 May 2013

Copyright (C) 2013 Jason B. Welch et al. This is an open access article distributed under the Creative Commons Attribution License, which permits unrestricted use, distribution, and reproduction in any medium, provided the original work is properly cited.

\begin{abstract}
Background: Detection of hepatocellular carcinoma (HCC) in high risk populations has traditionally involved the use of ultrasound (US), followed by computed tomography (CT) or magnetic resonance imaging (MRI) for confirmation of suspected lesions. The aim of our study was to determine, retrospecttively, in an unselected population of patients with cirrhosis, if an initial US could be as accurate as a subsequent CT or MRI in detecting HCC. Methods: 1600 consecutive cases from 2004-2007 in which US was performed for HCC screening were evaluated. 160 were identified which had an initial US followed by CT or MRI within 6 months. This group included 34 cases of HCC and 126 controls without HCC. Results: 26 cases of HCC were correctly identified by US, representing a sensitivity of $76.5 \%$; the mean alphafetoprotein (AFP) level was $440.4 \mathrm{ng} / \mathrm{dL}$. The other 8 cases had a false negative US; the mean AFP was 212.4 ng/dL. In 125 controls the mean AFP was 17.14 ng/dL. This group had only 12 patients, or $9.6 \%$, with an AFP greater than $20 \mathrm{ng} / \mathrm{dL}$, and only 1 who had an AFP greater than $400 \mathrm{ng} / \mathrm{mL}$. In this study, the positive predictive value of US for detecting HCC was $96.3 \%$, while the negative predictive value was $94.0 \%$. Combining US with the AFP level, and using a cutoff of $20 \mathrm{ng} / \mathrm{dL}$, the sensitivity was increased to $87.5 \%$. In this series of 160 patients, only 2 patients with HCC had a negative US and normal serum AFP. Conclusion: In combination with regular monitoring of AFP levels, US can eliminate more expensive imaging studies until further investigation by CT or MRI of questionable US results or elevated AFP is necessary, thus reducing the overall cost of monitoring these populations.
\end{abstract}

Keywords: Cirrhosis; Hepatitis C; Hepatitis B; Screening; Alpha-Fetoprotein

\section{INTRODUCTION}

Hepatocellular carcinoma (HCC) remains a leading cause of cancer death worldwide and an increasingly important cause of cancer related morbidity and mortality in the United States. One estimate places the total number of HCC cases per year in the United States between 8500 and 11,500 [1]. Incidence in the United States has been increasing for many years, with age-adjusted incidence rates tripling from 1975 to 2005 and overall mortality rates increasing at an annual percentage change of $1.6 \%$ [2]. In unscreened patients $\mathrm{HCC}$ is frequently detected when it is beyond treatment $[3,4]$. Thus, the search for an optimal screening program is important to find patients who have tumors small enough to be definitively treated.

AFP is a plasma protein encoded by the AFP gene on chromosome $4 \mathrm{q} 13.3$. It is the predominant plasma protein produced in the fetal liver and yolk sac and is believed to be the fetal equivalent of albumin. It has been associated with non-seminomatous germ cell tumors in males, gastric cancer, and neural tube defects in infants. It is also used in prenatal screening for open neural tube defects (increased), and trisomies 18 and 21 (decreased) [5-7].

Outcome of HCC is generally poor, as most cases present at an advanced stage $[3,4]$. Hence, screening of patients with the strongest risk factors for $\mathrm{HCC}$ is recommended, with the aim to detect $\mathrm{HCC}$ at an early stage and improve survival. In fact it has been shown that surveillance for $\mathrm{HCC}$, particularly in hepatitis B virus (HBV) infected patients, improves survival, as HCC detected by surveillance was more amenable to therapy [8]. 
Ultrasound (US) examination every 6 months is recommended by numerous practice guidelines [3,9-11]. Past study has shown that combined use of US and serum AFP can increase detection rates, but at the expense of increased false positives and thus increased overall cost of screening [12].

Patients who have abnormal US examinations with a suspicion for HCC are then followed with computed tomography (CT) or magnetic resonance imaging (MRI) for confirmation. It was our aim in this study to revisit the accuracy of US as the initial screening tool to determine if US combined with alpha-fetoprotein (AFP) should remain a starting point in evaluation of patients at risk for the development of HCC.

\section{METHODS}

\subsection{Permission and Approval}

Permission to undertake this retrospective review was given by the Institutional Review Board under their rules for collecting existing data given on March 12, 2009 in IRB number 09-066.

\subsection{Study Population}

A list of all patients consecutively undergoing a screening US examination of the liver (January 2004-May 2006) was obtained from the Radiology department. From this list, patients who had screening US and subsequent follow up CT or MRI within 6 months formed the study population. Patients with a pre-existing diagnosis of HCC were excluded. Patients who had both imaging studies on the same day were also excluded, as it was possible for the $\mathrm{CT}$ to be completed and/or reported prior to the US. Cases consisted of diagnosed HCC, while controls had no HCC.

\subsection{Definitions}

Cirrhosis was diagnosed using hematological, biochemical, and radiological criteria and liver biopsy was not required to be a necessary component for diagnosis of cirrhosis. HCC was defined using the standard AASLD guidelines with elevated AFP $>200$ and a lesion on US and/or the typical pattern of a focal lesion on triple phase CT scan [4].

\subsection{Data Collection}

Demographic (age, gender, race, etiology of cirrhosis, co-morbidities, and body mass index or BMI) and biochemical (hemoglobin, platelet count, serum aspartate aminotransferase or AST, serum alanine aminotransferase or ALT, AST/ALT ratio, alkaline phosphatase, total bilirubin, albumin, prothrombin time, serum creatinine, serum AFP) data at the time or within a week of US examination were recorded in a predefined excel spreadsheet. Child-Turcotte-Pugh (CTP) and Model for EndStage Liver Disease (MELD) scores were calculated for each patient using the standard formulae and scoring systems $[13,14]$. US imaging data were recorded as normal or abnormal. If there was no focal lesion on US, other abnormalities such as nodularity, heavy or increased echotexture, splenomegaly, and the presence of collateral vessels were recorded for each patient in the spreadsheet. CT or MRI imaging data were screened for abnormalities and recorded. The presence or absence of HCC after the CT/MRI was performed was recorded. Records of each patient included were reviewed by NI, RS and JW and all data compiled into a spread sheet. The data was reviewed by the group of investigators at the end of the collection period.

A serum AFP level was available in all but 1 of the HCC patients and in 86 of the 125 patients without HCC.

\subsection{Cost}

The study was performed with intramural funds and funding from the Marie B. Gale Centennial Professorship (RDS).

\subsection{Statistical Analysis}

Based on the findings of US and a final diagnosis of HCC after the CT/MRI, patients with diagnosed HCC were defined as true positive: focal lesion on US, and false negative: no focal lesion on US. Patients with no HCC were defined as true negative: no focal lesion on US, and false positive: focal lesion on US. Using these numbers, sensitivity, specificity, positive predictive value (PPV), and negative predictive value (NPV), of US examination were calculated. Sensitivity, specificity, PPV, and NPV were also calculated for US combined with AFP. For these calculations, all cases lacking an AFP measurement at the time of US were excluded. Within each group, cases were divided into three AFP categories: normal $(<20 \mathrm{ng} / \mathrm{dL})$, indeterminate $(20-400 \mathrm{ng} / \mathrm{dL})$, and abnormal for HCC diagnosis ( $>400 \mathrm{ng} / \mathrm{dL}$ ). When calculating these values, a true negative was required to have both a negative US and normal AFP. Conversely, a true positive was required to have both a positive US and AFP $>20 \mathrm{ng} / \mathrm{dL}$. Based on these calculations, an assessment was made as to how many CT scans could have been avoided and the rate of overutilization of CT scans was determined among the entire cohort of patients with cirrhosis who underwent screening US.

Once all data were compiled comparisons were made between: 1) The control (true negative) group and all HCC cases; 2) true negative and false negative cases; and 3) true positive and false negative cases. For these com- 
parisons, Fisher's exact tests and student's t-tests were used for categorical and continuous variables respectively. All statistical calculations were performed using Statistical Analysis Software (SAS) version 9.2 (SAS Inst. Cary, NC, USA). $P$ values $<0.05$ were considered significant.

\subsection{Evaluation of False Negatives}

At the completion of this study the false negative cases were reviewed in an attempt to identify features which could aid in detecting HCC lesions. US and CT/MRI reports were further examined in each of these cases to identify the types of HCC lesions that were not detected by US examination.

\section{RESULTS}

\subsection{US and/or AFP in the Detection of HCC}

Of the 1600 consecutive patients undergoing screening US, 160 met the inclusion criteria. A total of 34 HCC cases were detected. Of these, 26 were correctly diagnosed (true positive) while $8 \mathrm{HCC}$ cases were missed on the US examination (false negative). Of the 126 patients with no HCC (control group), 125 had no lesion on US (true negative) and 1 patient had a focal lesion on US (false positive) which was not seen on CT. Based on these numbers, US had a sensitivity of $76.5 \%$, specificity of $99.2 \%$, NPV of $94 \%$, and PPV of $96.3 \%$ for detecting HCC in this cohort of patients with cirrhosis. Accuracy of US for detecting HCC was $94 \%$ calculated as (true positives + true negatives)/total number of US examinations.

When using an AFP cutoff of $20 \mathrm{ng} / \mathrm{dL}$, combined use of US with AFP provided an increase in sensitivity to $87.5 \%$. Minimal increases in PPV, NPV, and specificity were also recorded in this group, as seen in Table $\mathbf{1 .}$ Similarly, using an AFP cutoff of $400 \mathrm{ng} / \mathrm{dL}$ yielded a sensitivity of $53.8 \%$, which was markedly decreased. The reason for this large decrease in sensitivity is directly related to the method for assignment of true positive and false negative values. Because the definition of false negative was changed in this setting to include an AFP of less than $400 \mathrm{ng} / \mathrm{dL}$, a much larger subset of false negatives were recorded.

Table 1. Sensitivity, specificity, Positive Predictive Value (PPV), and Negative Predictive Value (NPV) for US alone and in combination with different AFP upper limit.

\begin{tabular}{ccccc}
\hline & Sensitivity & Specificity & PPV & NPV \\
\hline US Alone & $76.47 \%$ & $99.21 \%$ & $96.3 \%$ & $93.9 \%$ \\
US + AFP $(<20 \mathrm{ng} / \mathrm{dL})$ & $87.50 \%$ & $100.00 \%$ & $100 \%$ & $97.3 \%$ \\
US + AFP $(<400 \mathrm{ng} / \mathrm{dL})$ & $53.85 \%$ & $100.00 \%$ & $100 \%$ & $93.4 \%$ \\
\hline
\end{tabular}

\subsection{Comparison of Cases and Controls}

The Comparison of demographic and laboratory data for these groups is found in Table 2. A significant difference was found between the HCC and control group for patient age, platelet count, and serum albumin level. HCC cases also had an increased percentage of male subjects compared to controls $(88.2 \%, 71.2 \% ; P=0.042)$. The overall mean for AFP in the study population was 114.69 $\pm 475.84 \mathrm{ng} / \mathrm{dL}$. The group mean AFP was also calculated for the control group $(17.14 \pm 53.26 \mathrm{ng} / \mathrm{dL})$, the false negative group $(212.37 \pm 411.57 \mathrm{ng} / \mathrm{dL})$, the true positive group $(440.39 \pm 411.57 \mathrm{ng} / \mathrm{dL})$, and all $\mathrm{HCC}$ $(\mathrm{TP}+\mathrm{FP})$ cases $(388.90 \pm 877.27 \mathrm{ng} / \mathrm{dL})$. Two outliers were present in the AFP measurements, with 1 value of $200,000 \mathrm{ng} / \mathrm{dL}$ in the true positive group, and the other of $257,115 \mathrm{ng} / \mathrm{dL}$ in the false negative group. Removing these two values, there was a significant difference in AFP levels between the control and HCC groups as well $(17.14,388.90 ; P=0.025)$.

Table 2. Comparison of controls vs. hepatocellular carcinoma cases.

\begin{tabular}{|c|c|c|c|}
\hline & Control group & All HCC cases & $\boldsymbol{P}$ \\
\hline Age (years) & $52.95 \pm 9.47$ & $58.97 \pm 9.03$ & 0.001 \\
\hline Hemoglobin $(\mathrm{g} / \mathrm{dL})$ & $12.23 \pm 2.63$ & $12.09 \pm 2.59$ & 0.778 \\
\hline Platelet count $\left(\times 10^{3} / \mu \mathrm{L}\right)$ & $131.86 \pm 83.37$ & $100.69 \pm 55.94$ & 0.015 \\
\hline AST & $118.68 \pm 177.47$ & $222.24 \pm 370.97$ & 0.123 \\
\hline ALT & $93.43 \pm 161.17$ & $84.88 \pm 54.77$ & 0.626 \\
\hline $\mathrm{AST} / \mathrm{ALT}$ ratio & $1.41 \pm 0.90$ & $2.39 \pm 2.98$ & 0.069 \\
\hline Alkaline phosphatase & $162.49 \pm 123.43$ & $202.41 \pm 159.38$ & 0.184 \\
\hline Total bilirubin & $2.65 \pm 4.12$ & $2.70 \pm 2.25$ & 0.947 \\
\hline Albumin & $3.22 \pm 0.89$ & $2.69 \pm 0.67$ & 0.001 \\
\hline Prothrombin time (s) & $16.24 \pm 5.06$ & $16.24 \pm 4.79$ & 0.997 \\
\hline CTP score & $7.14 \pm 2.20$ & $7.94 \pm 1.98$ & 0.054 \\
\hline MELD score & $13.01 \pm 8.10$ & $13.89 \pm 4.34$ & 0.414 \\
\hline AFP & $17.14 \pm 53.26$ & $388.90 \pm 877.27$ & 0.025 \\
\hline Race, $n(\%)$ & & & $\chi^{2}$ \\
\hline White & $65(52)$ & $19(55.9)$ & 0.923 \\
\hline AA & $22(17.6)$ & $5(14.7)$ & \\
\hline Hispanic & $37(29.6)$ & $10(29.4)$ & \\
\hline American Indian & $1(0.8)$ & 0 & \\
\hline Gender, $n(\%)$ & & & $\chi^{2}$ \\
\hline Male & $89(71.2)$ & $30(88.2)$ & 0.042 \\
\hline Female & $36(28.8)$ & $4(11.8)$ & \\
\hline Nodularity by US, $n(\%)$ & & & $\chi^{2}$ \\
\hline Yes & $48(38.4)$ & $19(55.9)$ & 0.173 \\
\hline No & $52(41.6)$ & $11(32.4)$ & \\
\hline Unknown & $25(20)$ & $4(11.8)$ & \\
\hline Coarse ET, $n(\%)$ & & & $\chi^{2}$ \\
\hline Yes & $93(74.4)$ & $30(88.2)$ & 0.224 \\
\hline No & $19(15.2)$ & $2(5.9)$ & \\
\hline Unknown & $13(10.4)$ & $2(5.9)$ & \\
\hline
\end{tabular}




\subsection{Comparison of Cases Missed on US with True Positives and True Negatives}

Comparison of the 8 cases missed on US examination (false negatives) with the 125 true negative cases showed that false negative cases were more likely to be older $(P-0.023$, Table 3$)$ with a trend toward higher AFP levels in the false negative group $(212.37 \pm 411.57,17.14 \pm$ 53.26; $P-0.256)$.

Comparison was also made between the false negative group and the true positive group, or cases correctly identified by US alone. In this comparison the only significant difference was a higher frequency of increased echotexture on US in the true positive group $\left(\chi^{2}-0.026\right.$, Table 4). Other notable differences between these two groups, while not statistically significant, were in the albumin level and the presence of nodularity on US $\left(P-0.063\right.$ and $\chi^{2}-0.094$, respectively).

Table 3. Comparison of controls vs. false negative cases.

\begin{tabular}{|c|c|c|c|}
\hline & Control group & False negatives & $\boldsymbol{P}$ \\
\hline Age (years) & $52.95 \pm 9.47$ & $61.00 \pm 11.41$ & 0.023 \\
\hline Hemoglobin (g/dL) & $12.23 \pm 2.63$ & $12.28 \pm 2.66$ & 0.969 \\
\hline Platelet count $\left(\times 10^{3} / \mu \mathrm{L}\right)$ & $131.86 \pm 83.37$ & $121.75 \pm 63.33$ & 0.679 \\
\hline AST & $118.68 \pm 177.47$ & $136.75 \pm 97.84$ & 0.646 \\
\hline ALT & $93.43 \pm 161.17$ & $93.00 \pm 61.23$ & 0.987 \\
\hline $\mathrm{AST} / \mathrm{ALT}$ ratio & $1.41 \pm 0.90$ & $1.49 \pm 0.67$ & 0.779 \\
\hline Alkaline phosphatase & $162.49 \pm 123.43$ & $207.88 \pm 216.07$ & 0.574 \\
\hline Total bilirubin & $2.65 \pm 4.12$ & $2.53 \pm 3.51$ & 0.925 \\
\hline Albumin & $3.22 \pm 0.89$ & $3.08 \pm 0.85$ & 0.643 \\
\hline Prothrombin time (s) & $16.24 \pm 5.06$ & $16.93 \pm 3.42$ & 0.612 \\
\hline CTP score & $7.14 \pm 2.20$ & $6.88 \pm 2.36$ & 0.735 \\
\hline MELD score & $13.01 \pm 8.10$ & $12.72 \pm 5.94$ & 0.900 \\
\hline AFP & $17.14 \pm 53.26$ & $212.37 \pm 411.57$ & 0.256 \\
\hline Race, $n(\%)$ & & & $\chi^{2}$ \\
\hline White & $65(52)$ & $4(50)$ & 0.964 \\
\hline AA & $22(17.6)$ & $1(12.5)$ & \\
\hline Hispanic & $37(29.6)$ & $3(37.5)$ & \\
\hline American Indian & $1(0.8)$ & 0 & \\
\hline Gender, $n(\%)$ & & & $\chi^{2}$ \\
\hline Male & $89(71.2)$ & $6(75)$ & 0.818 \\
\hline Female & $36(28.8)$ & $2(25)$ & \\
\hline Nodularity by US, $n(\%)$ & & & $\chi^{2}$ \\
\hline Yes & $48(38.4)$ & $2(25)$ & 0.511 \\
\hline No & $52(41.6)$ & $5(62.5)$ & \\
\hline Unknown & $25(20)$ & $1(12.5)$ & \\
\hline Coarse ET, $n(\%)$ & & & $\chi^{2}$ \\
\hline Yes & $93(74.4)$ & $6(75)$ & 0.525 \\
\hline No & $19(15.2)$ & $2(25)$ & \\
\hline Unknown & $13(10.4)$ & 0 & \\
\hline
\end{tabular}

Table 4. Comparison of true positive vs. false negative cases.

\begin{tabular}{|c|c|c|c|}
\hline & True positives & False negatives & $P$-value \\
\hline Age (years) & $58.35 \pm 8.33$ & $61.00 \pm 11.41$ & 0.557 \\
\hline Hemoglobin (g/dL) & $12.04 \pm 2.59$ & $12.28 \pm 2.66$ & 0.824 \\
\hline Platelet count $\left(\times 10^{3} / \mu \mathrm{L}\right)$ & $93.67 \pm 52.83$ & $121.75 \pm 63.33$ & 0.284 \\
\hline AST & $248.54 \pm 419.42$ & $136.75 \pm 97.84$ & 0.220 \\
\hline ALT & $82.38 \pm 53.69$ & $93.00 \pm 61.23$ & 0.668 \\
\hline AST/ALT Ratio & $2.67 \pm 3.35$ & $1.49 \pm 0.67$ & 0.102 \\
\hline Alkaline phosphatase & $200.73 \pm 142.98$ & $207.88 \pm 216.07$ & 0.932 \\
\hline Total bilirubin & $2.75 \pm 1.79$ & $2.53 \pm 3.51$ & 0.864 \\
\hline Albumin & $2.57 \pm .57$ & $3.08 \pm 0.85$ & 0.063 \\
\hline Prothrombin time (s) & $16.02 \pm 5.20$ & $16.93 \pm 3.42$ & 0.578 \\
\hline CTP score & $8.28 \pm 1.77$ & $6.88 \pm 2.36$ & 0.153 \\
\hline MELD score & $14.26 \pm 3.78$ & $12.72 \pm 5.94$ & 0.508 \\
\hline AFP & $440.39 \pm 411.57$ & $212.37 \pm 411.57$ & 0.375 \\
\hline Race, $n(\%)$ & & & $\chi^{2}$ \\
\hline White & $15(57.7)$ & $4(50)$ & 0.954 \\
\hline AA & $4(15.4)$ & $1(12.5)$ & \\
\hline Hispanic & $7(26.9)$ & $3(37.5)$ & \\
\hline American Indian & 0 & 0 & \\
\hline Gender, $n(\%)$ & & & $\chi^{2}$ \\
\hline Male & $24(92.3)$ & $6(75)$ & 0.184 \\
\hline Female & $2(7.7)$ & $2(25)$ & \\
\hline Nodularity by US, $n(\%)$ & & & $\chi^{2}$ \\
\hline Yes & $17(65.4)$ & $2(25)$ & 0.094 \\
\hline No & $6(23.1)$ & $5(62.5)$ & \\
\hline Unknown & $3(11.5)$ & $1(12.5)$ & \\
\hline Coarse ET, $n(\%)$ & & & $\chi^{2}$ \\
\hline Yes & $24(92.3)$ & $6(75)$ & 0.026 \\
\hline No & 0 & $2(25)$ & \\
\hline Unknown & $2(7.7)$ & 0 & \\
\hline
\end{tabular}

\subsection{False Negative Cases}

US and CT or MRI reports for the 8 false negative cases were reviewed in detail (Table 5). Tumors in this group were detected in varying numbers and sizes. The largest of these, $5.0 \times 3.3 \mathrm{~cm}$, was found on CT 3 months after US. Of note, this patient had multiple gallstones on US, a finding noted in 6 of the 8 cases. This patient's AFP was mildly elevated to 50 at the time of US, which should prompt further investigation alone. But, at the time of CT the AFP had already increased to 237, and eventually peaked at 18,833 1 year after the initial US. Other interesting findings in this group included a patient (Case 1, Table 5) with numerous tumors noted on CT, but no findings on US. In this patient a subsequent US also failed to detect the lesions 2 days after the CT. Two pa- 
Table 5. Characteristics of false negative cases.

\begin{tabular}{|c|c|c|c|c|c|c|c|}
\hline Case & Size on CT/MRI $(\mathrm{cm})$ & Other CT/MRI findings & $\begin{array}{l}\text { US to } \\
\text { CT/MRI time }\end{array}$ & MELD & CTP & AFP & Other US characteristics \\
\hline 1 & $\begin{array}{l}\text { Numerous, } \\
\text { size unknown }\end{array}$ & $\begin{array}{l}\text { PV thrombus to SMV, Distended } \\
\text { GB with calcifications }\end{array}$ & $24 \mathrm{~d}$ & 14 & 7 & 257115 & $\begin{array}{l}\text { CE, gallstones, normal PV flow; US } 2 \mathrm{~d} \\
\text { after CT unable to identify lesions }\end{array}$ \\
\hline 2 & $5.0 \times 3.3$ & $\begin{array}{l}\text { Cholelithiasis, invasion of right } \\
\text { hepatic vein }\end{array}$ & $3 \mathrm{~m}$ & 12 & 5 & 50 & $\mathrm{CE}$, distended $\mathrm{GB}$, multiple gallstones \\
\hline 3 & 1.5 & GB wall thickening, GB sludge & $20 \mathrm{~d}, 1 \mathrm{~d}$ & 25 & 12 & 19 & $\begin{array}{l}\text { Distended GB with sludge, LN, CE, } \\
\text { ascites, echogenic kidneys }\end{array}$ \\
\hline 4 & $\begin{array}{c}1.6 \times 1.8 \\
2.8 \times 2.6,<1 \mathrm{~cm}\end{array}$ & & $1 \mathrm{~d}$ & 16 & 6 & 65.3 & $\mathrm{CE}, 4 \mathrm{~mm}$ gallstone, $\mathrm{GB}$ wall thickening \\
\hline 5 & 3.6 & Ascites, dilated PV & $11 \mathrm{~d}$ & 11 & 8 & 1135.7 & $\mathrm{LN}, \mathrm{CE}$, ascites, dilated $\mathrm{CBD}$, absent $\mathrm{GB}$ \\
\hline 6 & $\begin{array}{l}2 \text { lesions, } \\
\text { size unknown }\end{array}$ & PV thrombosis, tumor extension & $3 \mathrm{~d}$ & 11 & 7 & 185.3 & $\begin{array}{l}\mathrm{LN}, \mathrm{CE}, \mathrm{PV} \text { thrombus, GB wall } \\
\text { thickening; repeat US at } 3 \mathrm{~d} \text { without } \\
\text { lesion, but thrombosed radical of PV }\end{array}$ \\
\hline 7 & 1.5 & Focal GB wall thickening & $6 \mathrm{~m}$ & 6 & 5 & 3.6 & $\begin{array}{l}\text { GB wall thickening with } 2 \text { areas of focal } \\
\text { thickening }\end{array}$ \\
\hline 8 & $3.6 \times 4.0,1$ & Numerous splenic granulomas & $2 \mathrm{~m}$ & 7 & 5 & 27.7 & \\
\hline
\end{tabular}

tients were found to have both negative US and normal AFP (Cases 3 and 7, Table 5). Both of these cases had features which would have led to further investigation. In Case 3, ascites and echogenic kidneys would be suspicious for more advanced disease. In Case 7, areas of gallbladder wall thickening would have likely prompted further study. In both of these cases a single lesion was identified on CT/MRI, each measuring $1.5 \mathrm{~cm}$.

\subsection{Cost Exploration}

It was determined that $74 \mathrm{CT}$ exams were performed on patients with both a negative US and normal AFP $(<20$ $\mathrm{ng} / \mathrm{dL}$ ). At this institution the estimated cost for an abdominal CT with contrast is $\$ 2359$, while a focused US of the right upper quadrant is only $\$ 555$. This is a difference of $\$ 133,496$ in this patient subset alone.

\section{DISCUSSION}

In a series of 1600 patients consecutively screened for hepatocellular carcinoma, of which 160 met inclusion criteria, sensitivity for the use of ultrasound alone was $76.5 \%$, while specificity, and positive and negative predictive values were all greater than $90 \%$. One previously reported problem with combining AFP and US was an increased false positive rate, which subsequently lead to increased screening costs [12]. While cost effectiveness was not addressed in our study, the false positive rate was only $0.8 \%$ for US alone, and decreased to $0 \%$ for combined US and AFP less than $20 \mathrm{ng} / \mathrm{dL}$. Current guidelines from the American Association for the Study of Liver Diseases recommend initial screening with US alone at an interval of 6 months in populations at risk for developing HCC [4]. These guidelines do not currently recommend for or against combined screening with AFP and US, but merely state that the increased sensitivity comes with an increased cost of screening [4].

\subsection{Evaluation of False Negatives}

In this study 8 patients were found to have HCC lesions by CT or MRI which were undetected on initial US in the preceding 6 month window. Of these 8 cases, 6 were noted to have coarsened liver echotexture by US, a common characteristic hindering the detection of hepatic masses.

In the entire study population, $123(76.9 \%$ of total, $85.4 \%$ of reported) patients were said to have coarsened echotexture or increased echogenicity. One might expect increased echotexture or coarsened echogenicity in a group of patients where HCC lesions were missed by US. After all, variable appearance of the liver parenchyma would reasonably be expected to cause difficulty in determining the presence or absence of any type of abnormality; however, this study group actually exhibited a greater incidence of increased echotexture in patients who were correctly diagnosed versus those missed by US [15]. In fact, the false negative subset of patients actually mimicked the control group with respect to liver echotexture, with nearly $75 \%$ of both subsets having increased echotexture, as opposed to $92 \%$ in the true positive HCC subset. The assumption that echotexture may play a role in disguising HCC lesions on US should be rejected, as it is a feature seen throughout this study population.

Another characteristic typically considered to complicate US interpretation is nodularity of the liver surface, the reason being that cirrhotic livers are capable of forming nodules large enough to be mistaken for masses [15]. Compared to the true positive group, the false negative group had a smaller percentage of patients with nodular- 


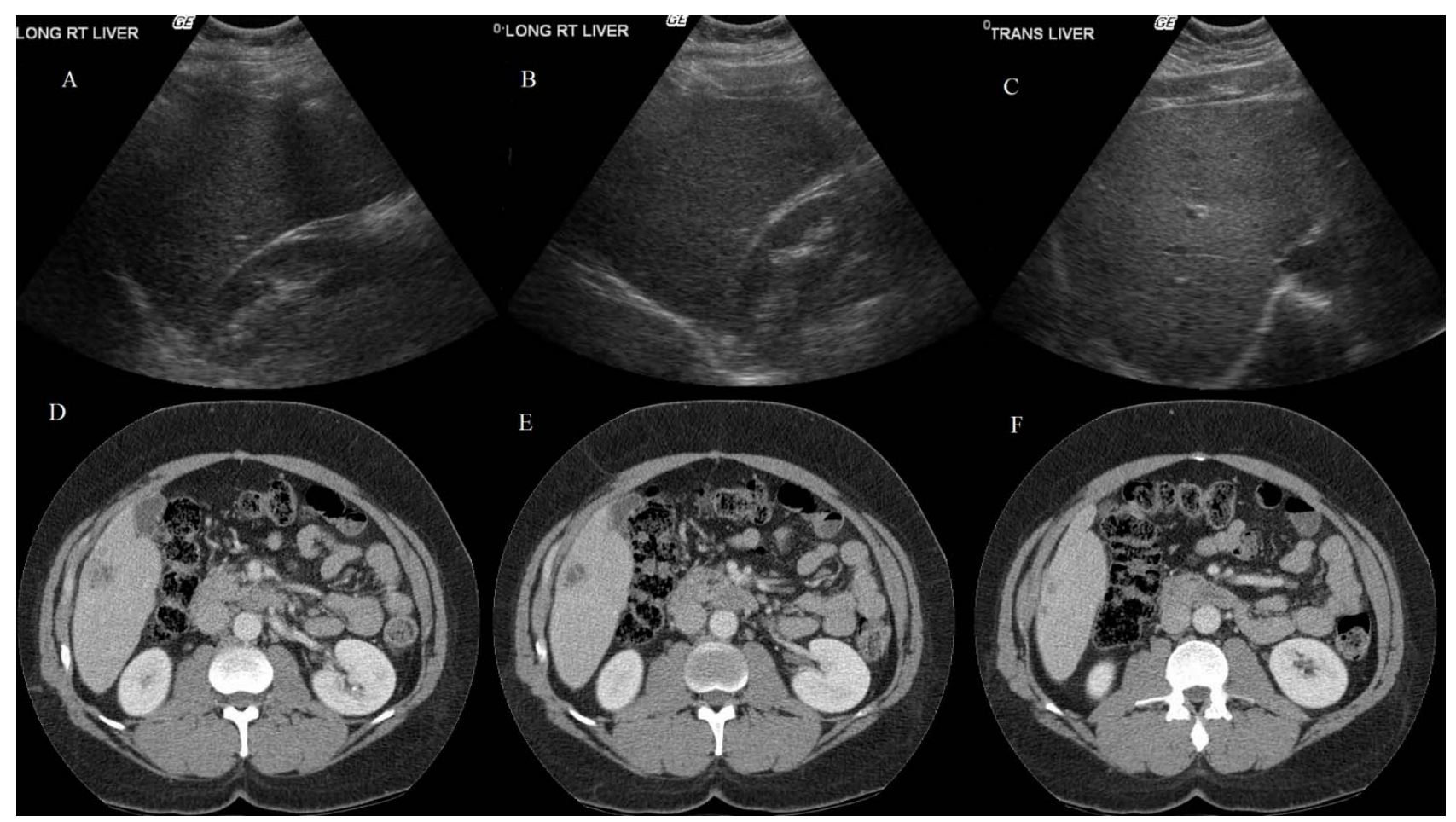

Figure 1. Right lobe long ((A), (B)) and transverse (C) views accompanied by consecutive CT images showing a single large hypodense lesion ((D), (E)) with an adjacent smaller lesion (F), all in liver segment VI.

ity; however, this difference was not statistically significant $(65.4 \%, 25 \% ; P=0.094)$. As is the case with echotexture, it would appear that the presence of increased surface nodularity should not be considered a feature which will disguise HCC lesions on US.

Although review of imaging in each case was not always possible, we were able to compare US and CT/MRI images in a few of these 8 cases. US exams at our institution are done by US technicians, who then record common US views as well as any obvious abnormalities on exam. These images are then interpreted by a radiologist who files a dictated report in the electronic medical record (EMR). This allows for the possibility of missing masses on US which may be more obvious on CT, as occurred in several instances above (Cases 2, 5, 8; Table 5). This is demonstrated in Figure 1, where consecutive CT images from Case 8 (Table 5) show a hypodense segment VI lesion of substantial size. The accompanying static US images, however, fail to capture any of this mass in the right hepatic long or transverse views.

\subsection{Cost Exploration}

While specific data regarding cost were not collected for each individual imaging exam, it is clear that avoiding unnecessary CT or MRI would be beneficial from a financial perspective as well. In this population of 160 patients an estimated $\$ 133,496$ could potentially have been saved in this manner.

\subsection{Limitations}

One limitation of this study was its retrospective nature. Because no persons involved were aware that US and CT/MRI reports would later be used for research, there were often differences in reporting styles among interpreting physicians. This made analysis of tumor size data impossible, as some reports were lacking measurements of tumor size on both imaging techniques. Differences in reporting style also contributed to the difficulty in comparing reports.

\section{CONCLUSION}

US is an effective tool for detection of HCC. Despite increasingly advanced methods for detecting hepatic lesions, which will undoubtedly improve screening in the future, our findings suggest that the use of US and AFP is an appropriate method and should remain the first step for HCC screening in patients at an increased risk for the occurrence of hepatocellular carcinoma.

\section{REFERENCES}

[1] El-Serag, H.B. (2004) Hepatocellular carcinoma: Recent trends in the United States. Gastroenterology, 127, S27S34. doi:10.1053/j.gastro.2004.09.013

[2] Altekruse, S.F., McGlynn, K.A. and Reichman, M.E. (2009) Hepatocellular carcinoma incidence, mortality, and survival trends in the United States from 1975 to 2005. 
Journal of Clinical Oncology, 27, 1485-1491. doi:10.1200/JCO.2008.20.7753

[3] Bruix, J. and Sherman, M. (2010) Management of heaptocellular carcinoma: An update, AASLD practice guidelines. Hepatology, 53, 1-3.

[4] Llovet, J.M., Burroughs, A. and Bruix, J. (2003) Hepatocellular carcinoma. Lancet, 362, 1907-1917. doi:10.1016/S0140-6736(03)14964-1

[5] Liu, X., Cheng, Y., Sheng, W., et al. (2010) Clinicopathologic features and prognostic factors in alpha-fetoprotein-producing gastric cancers: Analysis of 104 cases. Journal of Surgical Oncology, 102, 249. doi:10.1002/jso.21624

[6] Gilligan, T.D., Seidenfeld, J., Basch, E.M., et al. (2010) American Society of Clinical Oncology Clinical Practice Guideline on uses of serum tumor markers in adult males with germ cell tumors. Journal of Clinical Oncology, 28, 3388. doi:10.1200/JCO.2009.26.4481

[7] Driscoll, D.A. and Gross, S.J. (2009) Professional Practice Guidelines Committee. Screening for fetal aneuploidy and neural tube defects. Genetics in Medicine, 11, 818. doi:10.1097/GIM.0b013e3181bb267b

[8] Tong, M.J., Sun, H.-E., Hsien, C., et al. (2010) Surveillance for hepatocellular carcinoma improves survival in Asian-American patients with hepatitis B: Results from a community-based clinic. Digestive Diseases and Sciences, 55, 826-835.

[9] Makuuchi, M., Kokudo, N., Arii, S., et al. (2008) Devel- opment of evidence-based clinical guidelines for the diagnosis and treatment of hepatocellular carcinoma in Japan. Hepatology Research, 38, 37-51. doi:10.1111/j.1872-034X.2007.00216.x

[10] Kudo, M. (2010) The 2008 okuda lecture: Management of hepatocellular carcinoma: From surveillance to molecular targeted therapy. Journal of Gastroenterology and Hepatology, 25, 439-452. doi:10.1111/j.1440-1746.2009.06207.x

[11] Bruix, J., Sherman, M., Llovet, J.M., et al. (2001) Clinical management of hepatocellular carcinoma. Conclusions of the Barcelona-2000 EASL Conference. Journal of Hepatology, 35, 421-430. doi:10.1016/S0168-8278(01)00130-1

[12] Zhang, B. and Yang, B. (1999) Combined alpha fetoprotein testing and ultrasonography as a screening test for primary liver cancer. Journal of Medical Screening, 6, 108-110.

[13] Kamath, P.S., Wiesner, R.H., Malinchoc, M., et al. (2001) A model to predict survival in patients with end-stage liver disease. Hepatology, 33, 464-470. doi:10.1053/jhep.2001.22172

[14] Child, C.G. and Turcotte, J.G. (1964) Surgery and portal hypertension. Major Problems in Clinical Surgery, 1, 185.

[15] Nguyen, M. and Keeffe, E. (2002) Screening for hepatocellular carcinoma. Journal of Clinical Gastroenterology, 35, S86-S91. doi:10.1097/00004836-200211002-00004 